This is an accepted manuscript. The published version may differ from it. Please cite as: Singh, M. (forthcoming) Subjective selection and the evolution of complex culture. Evolutionary Anthropology.

\title{
Subjective selection and the evolution of complex culture
}

\author{
Manvir Singh \\ Institute for Advanced Study in Toulouse, Université de Toulouse 1 Capitole, Toulouse, France \\ manvirsingh@iast.fr
}

\begin{abstract}
Why is culture the way it is? Here I argue that a major force shaping culture is subjective (cultural) selection, or the selective retention of cultural variants that people subjectively perceive as satisfying their goals. I show that people evaluate behaviors and beliefs according to how useful they are, especially for achieving goals. As they adopt and pass on those variants that seem best, they iteratively craft culture into increasingly effective-seeming forms. I argue that this process drives the development of many cumulatively complex cultural products, including effective technology, magic and ritual, aesthetic traditions, and institutions. I show that it can explain cultural dependencies, such as how certain beliefs create corresponding new practices, and I outline how it interacts with other cultural evolutionary processes. Cultural practices everywhere, from spears to shamanism, develop because people subjectively evaluate them to be effective means of satisfying regular goals.
\end{abstract}

Keywords: adaptation, cognition, cultural evolution, culture, technology 
Where do igloos come from? And spears? And rain magic? And techniques for processing manioc? Where does bloodletting come from? And shamanism? Why are there chairs? Why are there hammers?

These questions are all specifications of a foundational question: What explains the design of culture? Here, I argue that an important answer is subjective (cultural) selection, or the selective retention of practices and beliefs people subjectively evaluate as useful, especially for achieving goals. Traditions as diverse as spears, hero tales, shamanism, witchcraft beliefs, and food-processing all evolve because they appear to satisfy people's motivations.

There are two reasons I refer to this process as 'subjective'. The first is that people's goals are subjective. They are determined by their psychology. People want retribution. They crave pleasure. They want food and sex and prestige. The practices they build appease these and other goals. It's true that, in satisfying these goals, people often enjoy objective benefits. But in many cases, they pursue goals with neutral, even negative, effects. They watch pornography, eat cheesecake, and smoke cigarettes, often without fitness or material advantages.

The second reason I use the word 'subjective' is that humans subjectively evaluate culture. Sometimes we're good at this evaluation. I can tell whether a match produced a fire, whether a spear hit its target, and whether a cookie tastes good. But other times, our evaluations are mistaken. We think that divination works, that spells invite rain, and that blowing on dice might bump our chances of a lucky roll. Explaining culture requires considering both the goals human pursue and how they evaluate whether those goals are achieved.

Various researchers in the mid- to late- $20^{\text {th }}$ century emphasized the importance of goals and subjective evaluation in guiding the evolution of culture. ${ }^{1}$ Boehm proposed that variation, selection, and retention are constrained by goal-driven foresight: We want to solve particular problems, and this determines how we create and choose culture. ${ }^{2}$ Ruyle pointed out that people seem motivated to satisfy drives, such as for air, food, clothing, and water. ${ }^{3}$ On that basis, he argued, individuals will adopt and pass on those cultural variants that best satisfy their drives, fueling the evolution of culture. Boyd and Richerson suggested that people might use the attractiveness of a variant to determine whether they should adopt $\mathrm{it}^{4}$ — what they called 'direct bias' and what others have since termed 'content bias'. ${ }^{5}$ Such biases, they wrote, include evaluations of how well a variant seems to achieve a goal. They also posited that, through individual learning, people preferentially adopt behaviors which satisfy "objectives or guiding criteria" - a process they called 'guided variation'.

Despite these promising directions, the roles of goals and evaluation in cultural evolution have remain underdeveloped in contemporary cultural evolution research. Instead, scholars focus on other hypothesized processes, such as those that maximize individual- or group-level success or that select for memorable or attention-grabbing variants. ${ }^{4,6-8}$ The result is that many researchers draw a partition among cultural evolutionary theories, isolating theories well-suited to explain useful technology from those that are useful for explaining symbolic and expressive culture. ${ }^{9-11}$ This dichotomy in process is echoed by a common distinction between cultural "adaptations", on the one hand, and "by-products", on the other.

In this paper, I show that this distinction is often unnecessary-that, aside from being by-products or adaptations, practices of all types are subjectively compelling solutions. From kayaks to stories to magic to institutions, a diversity of cultural practices evolve because they satisfy our evaluation criteria for achieving desired ends. 


\section{Subjective cultural selection}

As people preferentially produce and evaluate cultural variants, they select those that best satisfy psychologically determined criteria. These are typically the variants that appear to best achieve a goal, from killing a monkey to feeling pleasure to explaining misfortune. Over time, this iterative selection drives the evolution of culture shaped to apparently appease regular goals.

There are at least two critical points here. First is the emphasis on subjective rather than objective efficacy. It doesn't matter whether a variant actually promotes individual- or group-level success. Rather, what's important is that individuals judge a variant to be useful (or, more precisely, that the variant satisfies psychological criteria for adopting behaviors or beliefs). Of course, subjective and objective efficacy will often converge. A person hoping to hunt animals might compare different spears and judge the more effective spear to be the better one. A person trying to hammer nails might choose the hammer that does the best job. In these instances, culture should evolve to become increasingly effective, and it is the process of iterative trial and error that often drives the evolution of so-called "adaptive" culture, such as useful technological toolkits. But, as I discuss later, subjective selection can also produce compelling but ineffective cultural practices, such as rain magic or futile money managers.

The second point is that culture must be produced, and producers need reasons. As André and colleagues ${ }^{12}$ recently argued, cultural practices survive only as long as people invest in them. Stories need to be told. Hammers need to be assembled. Shamans need to undergo initiations. Explaining these and other traditions requires understanding not only the practices' function or appeal, but the reasons individuals bother investing in them, as well. Subjective selection explains the design of culture as well as a critical dimension of its maintenance.

\section{Psychological foundations}

\section{What are goals, exactly?}

A 'goal' is an outcome towards which an individual is motivated. It can range from butchering a monkey to getting revenge. Although scholars long assumed that goal pursuit was conscious, or that it originated in conscious will, a large body of research now shows that people are often unaware of their goals and how their goals influence their behavior. ${ }^{13,14}$

Some scholars draw a conceptual distinction between two sets of goals. ${ }^{15,16}$ The first setincluding food, sex, trust, revenge, security, social status, and information — can be thought of as superordinate goals. Upon achieving these ends, humans and other organisms experience a hedonic signal, known as reward, characterized by activity in pleasure centers. ${ }^{15,16}$ That these superordinate goals guide our behavior reflects their adaptive value during our evolutionary history: Natural selection seems to have designed human psychology, and that of many other animals, to pursue outcomes that were reliably correlated with higher reproductive success. ${ }^{17}$

The second set comprises subordinate goals. These are hierarchically structured under superordinate goals. ${ }^{15,16}$ To get food, for instance, we might enact the following sequence of subgoals: Trek into forest, search for monkey, shoot monkey, climb tree, bring monkey down, butcher monkey, and so on. Because of the hierarchical nature of motivational behavior, everyday behavior can sometimes appear divorced from superordinate goals. For instance, the goal check email (and its components like open laptop and move cursor to browser icon) might eventually be in service of 
superordinate goals like secure social status and eat food, but with an elaborate hierarchy of goals connecting them. Just as we experience reward when we attain superordinate goals, the achievement of sub-goals seems to evoke a corresponding signal, known as pseudo-reward. ${ }^{15,16}$

\section{Humans select goal-directed behaviors through model-free and model-based evaluations}

How do we select behaviors (including sub-goals) when pursuing goals? Many behavioral researchers point to two systems of evaluation: habit and planning. ${ }^{18,19}$ In habit-known in a reinforcement learning framework as model-free learning - the organism selects those actions or action-sequences that previously led to a rewarding outcome (e.g., status, sex, food). This strategy is said to be "model-free" because the organism doesn't choose actions using a causal model of the environment, but instead stores the expected rewards, or values, of behaviors, updating those values through error-driven learning. For instance: I want an apple. I climb a tree. I fall and hurt myself. I integrate the new experience of falling with my previously computed value of climb tree, resulting in a lower expected reward.

In planning, in contrast, the organism selects actions or sequences of sub-goals by simulating them in a causal model of the environment. ${ }^{19}$ The organism has a desired end-state, like getting an apple, and then mentally simulates different actions and compares them. Known in a reinforcement learning framework as model-based learning, this strategy is more flexible than model-free learning but more computationally costly. ${ }^{20}$ As I discuss later, humans' causal models are shaped by culturally transmitted beliefs, and thus which behaviors they regard as valuable will vary with their cultural environment. Critically, both model-free and model-based learning seem to occur hierarchically: Individuals select and evaluate not only individual actions (like shoot monkey with gun) but goal-organized sets of actions, too (like hunt monkey). ${ }^{16}$

Behavioral and neuroscientific research provides evidence that people represent and track the payoffs of a hierarchy of goals. ${ }^{16,21,22}$ In one experiment, Ribas-Fernandes et al. ${ }^{23}$ presented participants with a computerized task in which they were rewarded for using a virtual truck to pick up a package and deliver it to a house. Hypothesizing that participants would represent picking up the package as a sub-goal (associated with pseudo-reward) and delivering the package as a superordinate goal (associated with reward), the experimenters unpredictably varied the ease of picking up the package while keeping the total distance time constant. As predicted, they found that trials in which the package was unexpectedly easier or harder to pick up triggered activity in regions associated with reward prediction error (as people presumably updated the value of the sub-goal), while in behavioral experiments, participants showed no overall preference for closer or more distant packages as long as the travel time was constant. In other words, participants appeared to track the value of a rewarding, overarching goal (delivering the packaging), while also representing and updating the values of sub-goals hierarchically organized under it. Diuk et al. ${ }^{24}$ found convergent results in an experiment in which participants played with, and chose among, virtual slot machines organized within casinos. People represent the costs and benefits of a hierarchy of goal-directed actions, using expected payoffs to structure decision-making.

\section{Social learning is constrained by individual evaluation}


A third process for selecting behavior is social learning. Numerous animal taxa engage in social learning, ${ }^{25}$ including insects ${ }^{26,27}$. Yet humans are uniquely reliant on and competent at social learning..$^{6,28,29} \mathrm{We}$ are equipped with adaptive learning biases, relying more on social information, for instance, when individual information is deficient ${ }^{30}$ and when behaviors are performed by many demonstrators (conformist bias) or especially successful ones (success or prestige bias). . $^{4,31}$ Still, that humans socially learn does not mean that any behavior can spread through a population. Instead, social learning seems constrained by individual evaluations. In his study of the diffusion of innovations, Rogers reviewed an extensive body of research examining why particular variants spread, covering innovations as diverse as hybrid seed-corn, boiling water, computer keyboards, solar panels, and steel axes among Aboriginal Australians. ${ }^{32} \mathrm{He}$ concluded that five characteristics are most predictive of whether an innovation spreads. Each reflects instrumental evaluation:

1. Relative advantage, or "the degree to which an innovation is perceived as better that the idea it supersedes"

2. Compatibility, or "the degree to which an innovation is perceived as being consistent with the existing values, past experiences, and needs of potential adopters"

3. Complexity, or "the degree to which an innovation is perceived as difficult to understand and use"

4. Trialability, or "the degree to which an innovation may be experimented with on a limited basis"

5. Observability, or "the degree to which the results of an innovation are visible to others"

These findings are echoed by research highlighting the role of evaluation in cultural transmission. Theoretically, several studies have shown the value of individual evaluation combined with social learning. ${ }^{33-35}$ Enquist and colleagues, for instance, found that a strategy of "critical social learning", or socially learning behaviors and then individually testing them, outcompetes pure social learning and is an evolutionary stable strategy except under limited circumstances. ${ }^{33}$ These theoretical predictions have been supported empirically. Heyes synthesized research in humans and non-humans indicating substantial involvement of nonsocial learning mechanisms (e.g., evaluation) in social learning. ${ }^{36}$ Olsson et al. reviewed neurobiological evidence that social learning often involves vicarious evaluation. ${ }^{37}$ Mice and primates both update the value of stimuli and behaviors on the basis of others' environmental feedback, and social learning recruits many networks central to individual learning-networks involved in predicting the expected outcome of a behavior and updating its value contingent on error. ${ }^{37}$ Finally, learners exhibit what Csibra and Gergely ${ }^{38}$ call an "obsession with goals". Just as children preferentially learn from individuals who resemble them or who are proficient, ${ }^{39}$ they also pay close attention to a demonstrator's instrumental ends when adopting behaviors. ${ }^{40}$ Despite suggestions of an inflexible or blind learning process-especially from studies of behaviors like over-imitation ${ }^{41}$ (although see refs. ${ }^{42,43}$ )—experimental and theoretical work indicates that evaluation is a major component of social learning. Behaviors and technologies can spread through social learning, but cognitive mechanisms involved in learning select for apparently useful, goal-directed behaviors. ${ }^{44}$ 


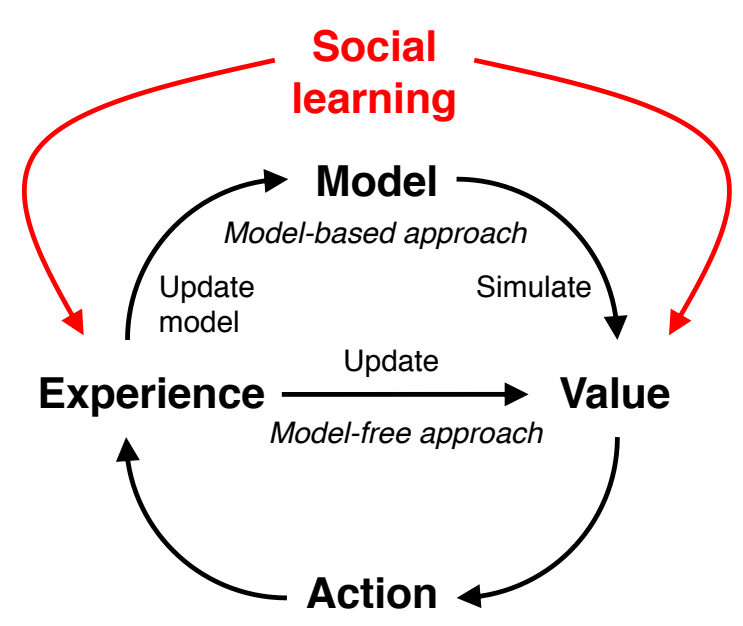

Figure 1. People adopt and continue to use a goal-directed behavior as long as it is perceived to be valuable. An individual evaluates a goal-directed behavior either by simulating it in a causal model of the environment (model-based approach) or by updating the value of a behavior after engaging in it (model-free approach). This evaluation ends with a computed value of the behavior, which determines whether the individual uses it (action). After acting, the individual again evaluates whether the behavior achieved the expected end, updating causal models and stored values accordingly. Individuals socially learn, but they evaluate learned beliefs and behaviors both before adopting them and after using them.

\section{Explaining culture}

As many scholars point out, the explanatory power of a cultural evolutionary framework hinges on its capacity to explain both adaptive and maladaptive cultural products. ${ }^{6,45,46}$ In that vein, I here show that subjective selection can explain a diversity of complex culture. It explains the origins of adaptive, complex technologies. It explains inefficiencies of otherwise adaptive systems or practices. It explains ubiquitous but ineffective technologies. It explains the development of many aesthetic traditions. And it can explain a variety of institutional outcomes, including group-functional rules, extractive rules, and sub-optimal persistence.

\section{Explaining the evolution of functional complexity}

According to the framework laid out here, useful technology develops as people subjectively evaluate whether variants satisfy their goals, crafting them to become increasingly effective. For example, a person can gauge whether a tweak to a spear allows it to fly further, keeping the tweaks that seem to work. As people repeat this process, spears become better at killing animals. This cumulative evolution can occur through individual trial-and-error, as a single person iteratively improves a solution, as well as across individuals, as people adopt and transmit the variants that seem to best achieve their aims (for a recent review on how demography impacts cumulative cultural evolution, see ref. ${ }^{47}$ ). Even in circumstances where people are less willing to produce innovations, such as in some very 'tight' cultures, ${ }^{48}$ they should still encounter variation through mistakes that, in turn, feeds subjective selection. 
This explanation differs from accounts that focus on the objective benefits of these technologies, such as those that link the cultural evolution of adaptive technology to natural selection (people with fitness-improving culture have more offspring) or iterated, cue-biased transmission (e.g., through imitating healthy individuals, practices that promote health spread through a population; see ref. ${ }^{49}$ for an example). Instead, what's key is that, through their subjective evaluations, people build efficient technologies. If people's subjective evaluations are systematically biased or erroneous, however, the resulting technologies should have corresponding inefficiencies, a prediction I address in the next section.

Can people really build increasingly effective solutions as they tweak, evaluate, and retain practices to satisfy their goals? Experimental research demonstrates that they can. In a recent study, Allen et al. presented participants on Amazon Mechanical Turk with a 2D virtual world and asked them to use tools to get a red ball into a green region. ${ }^{50}$ Through rapid trial-anderror-usually within 5 to 10 attempts-participants discovered how to use the novel objects to achieve their goal (see also McCarthy et al.'s study of how participants quickly learn how to build block towers ${ }^{51}$.

Most striking about Allen et al.'s results was that the authors modeled, using what they called the 'Sample, Simulate, Update' (SSUP) model, how participants discovered solutions. Guided by research on the so-called 'intuitive physics engine ${ }^{52}$, the SSUP model assumes that agents have an intuitive physical model of the world which guides their search process. Agents simulate actions within that causal mental model—essentially using model-based learning to devise a way of using a tool-before deploying an action. They evaluate how effective the action was for attaining their goal, updating their beliefs about the value of actions before choosing a new action. The SSUP model not only achieved a similar solution rate as human participants, but it discovered solutions after a similar number of trials. Although the SSUP model is a first approximation of the cognitive mechanics of trial-and-error learning, it demonstrates experimentally how subjective selection drives the evolution of functional cultural solutions. Technology becomes increasingly effective as individuals iteratively produce and evaluate goaldirected variants.

Allen et al.'s study focused on individuals working alone, but a large body of research has demonstrated subjective selection occurring across individuals. Across studies, sequences of participants cumulatively build complex culture as they tweak, evaluate, and selectively adopt practices that seem to best achieve their aims. ${ }^{53}$ For example, Zwirner and Thornton created experimental chains in which participants were told to produce baskets to hold as much rice as possible. ${ }^{54}$ Each participant worked on their basket and tested it before their solution was transmitted to the next participant. In addition to a solitary trial-and-error condition, the experimenters created three treatments: emulation, in which participants saw preceding individuals' baskets and learned about their success; imitation, in which they watched the previous participant build their basket and try it out; and teaching, in which participants were asked to help the next person after testing their basket. Across all of the treatments, including solitary trial-and-error, participants produced iteratively better baskets. They gauged how well the previous subject did and made changes accordingly. They even responded strategically to failure: Participants who observed a poorly performing basket were more likely to try to include new materials afterwards. This study and many others like it demonstrate that cumulative, effective culture can evolve as individuals selectively evaluate and retain modifications according 
to how well they seem to achieve a goal. ${ }^{55-57}$ Cumulative cultural evolution occurs as people keep what seems to work and drop what doesn't.

\section{Explaining cultural inefficiencies}

Subjective selection explains not only why culture evolves to be effective, but also why it is sometimes suboptimal. Specifically, it explains three classes of suboptimality.

First, subjective selection explains why some practices are effective yet suffer from systematic inefficiencies. As long as people have erroneous biases in how they evaluate variants, the resulting products should exhibit corresponding inefficiencies. Take competitive sports teams. ${ }^{58}$ Until the mid-2000s, the managers of Americans professional sports teams relied extensively on their individual evaluations of players when building teams. Those evaluations were useful but flawed. In baseball, for instance, people overvalued easy-to-observe traits, such as footspeed, while undervaluing traits that seemed less relevant to athleticism (e.g., a player's ability to have bad pitches thrown at them). ${ }^{58}$ The result was that all teams were sub-optimally constructed. Even in a context with high economic stakes and intense competition, people's flawed evaluation criteria lead to systematic inefficiencies in goal-directed products.

Second, subjective selection explains why people use compelling but ineffective practices. Comprising various kinds of superstition, political punditry, money management, and even medicine, ineffective practices are ubiquitous. Under a subjective selection framework, these practices succeed because of systematic biases in how we evaluate usefulness. When evaluating an action, we consider its cost, the benefit of the desired outcome, and the probability of the outcome. ${ }^{59}$ This leads to a kind of bet-hedging strategy: As long as a behavior appears to sometimes result in a desired outcome, and as long as it is cheap enough, people are predisposed to adopt it. ${ }^{60}$ For example, if a person desperately wants rain, and then a rainstorm serendipitously arrives, they are predisposed to look for low-cost actions that may have been responsible. Because of these biases, humans adopt useful but noisy behaviors (like avoiding foods that occasionally cause terrible illness) as well as ineffective actions ("superstitions") targeted at highly desired outcomes. Although researchers have argued that various superstitious or apparently ineffective practices may influence success or promote other functional benefits, ${ }^{61-63}$ such objective benefits are not necessary to explain these apparently ritualistic practices. Rather, they can develop and survive simply because they are effective-seeming. Box 1 demonstrates how this logic can be applied to explain shamanism, a longstanding anthropological puzzle.

Box 1. Shamanism as a product of subjective selection 


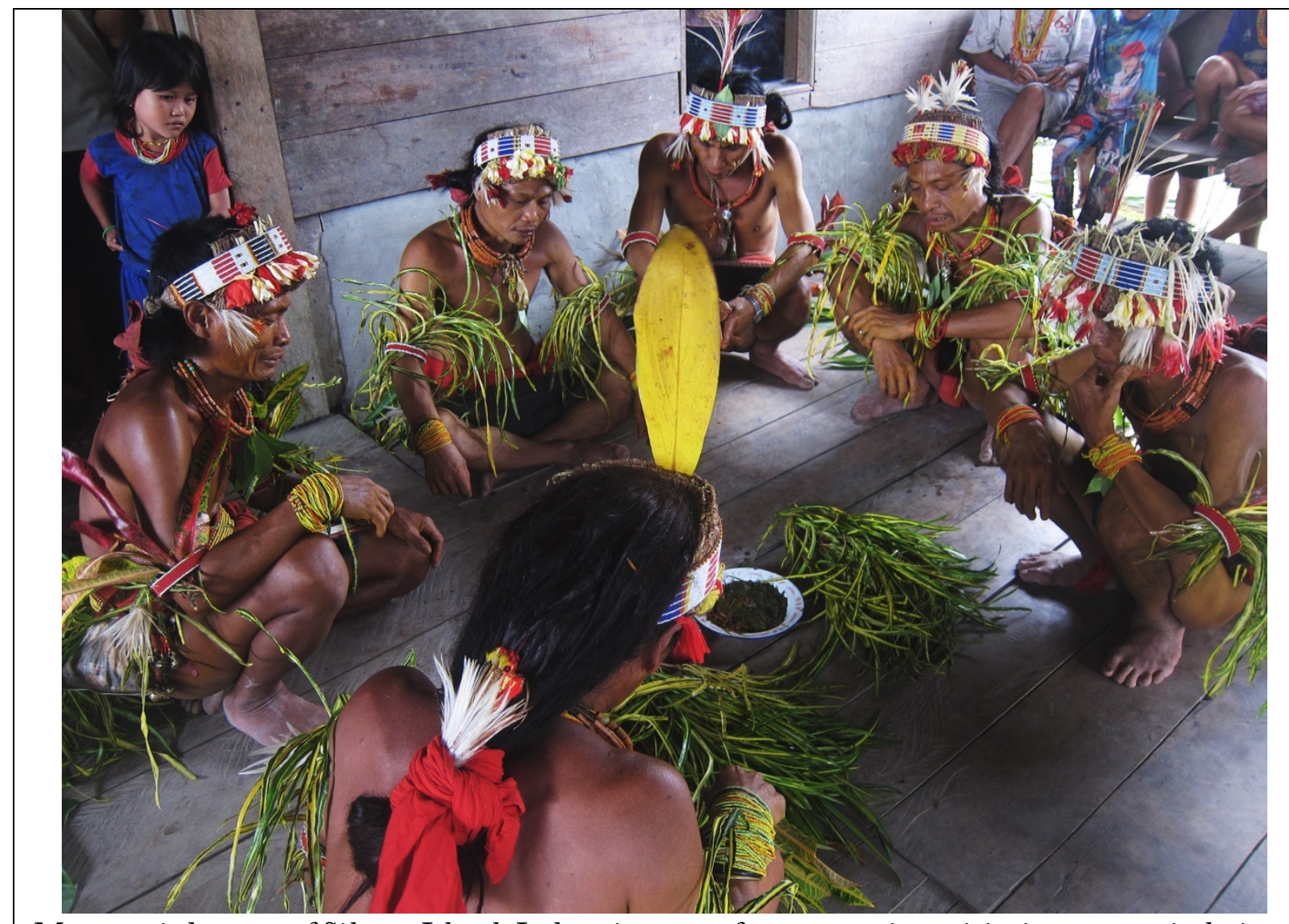

Mentawai shamans of Siberut Island, Indonesia weep after summoning spirits in a trance-inducing dance.

Shamans are practitioners who, in apparently foreign psychological states (trance), engage with invisible agents to provide services like healing and divination. ${ }^{64}$ Manifesting as mediums, channelers, witch-doctors, and the prophets of religious movements, shamans appeared in most human societies, ${ }^{65}$ including among nearly all documented hunter-gatherers. ${ }^{66}$ Yet despite shamanism's ubiquity, and its status as a central puzzle of anthropology, its origins have remained a mystery.

According to the subjective selection framework, shamanism may be an effectiveseeming way of influencing uncontrollable events. ${ }^{64}$ People everywhere try to influence important (roughly, fitness-relevant) events that randomly resolve, such as recovering from illness. Given the importance of these outcomes, and that they sometimes resolve in people's favor, people are predisposed to evaluate erroneous techniques as effective. ${ }^{67}$ As specialists compete to provide the most compelling magical services, and as people choose the most effective-seeming techniques, they jointly drive a subjective selection for compelling ways of influencing uncertainty, resulting in shamanism. In contrast to other accounts, ${ }^{68}$ this subjective model of shamanism predicts that practitioners will overwhelmingly assist with uncertain outcomes and less frequently act as leaders. As predicted, shamans across 43 diverse societies ubiquitously claim to help or provide information about uncertain outcomes (filled black bars in the accompanying figure) while less often assisting with life cycle activities (striped bars) or serving as leaders (empty bars). ${ }^{64}$ 


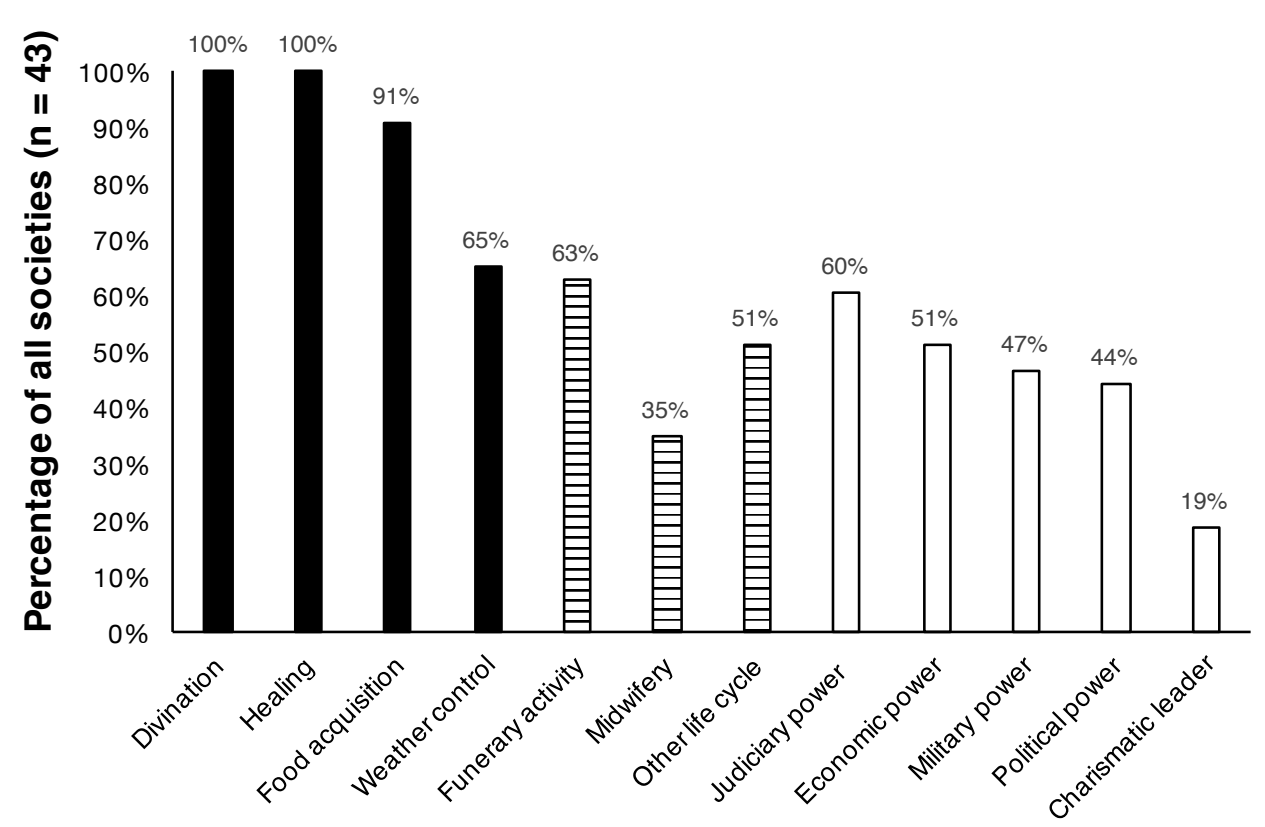

What makes shamans so compelling? Shamans use trance and initiations to apparently transform into entities distinct from normal humans. ${ }^{64}$ Like Superman's alien origins and the $\mathrm{X}$-Men's genetic mutations, these transformations assure people that shamans deviate from normal humans, making it more reasonable that they can engage with the invisible agents believed to oversee uncertain outcomes. In other words, core features of shamanism seem to recur because they promote the subjective perception that shamans are compelling and credible means of dealing with uncertain outcomes. This interpretation is supported by recent field experiments on shamanic self-denial among the Mentawai of Indonesia. ${ }^{69}$

The example of shamanism illustrates how elaborate, widespread traditions might reliably develop because they appear to satisfy regular goals. Shamanism also demonstrates how culturally transmitted causal models determine which features appear most effective. Shamans' defining practice - their use of trance to engage with supernatural beings - seems to reflect the widespread belief that invisible forces like gods and spirits determine uncertain outcomes. ${ }^{70}$ A prediction of the subjective model of shamanism is that, as people's beliefs change about what causes uncertain outcomes, shamanism should transform as well.

Last, subjective selection helps explain why some useful or effective practices fail to emerge or spread. Simply, useful practices are much less likely to develop when it's harder to gauge that they're effective. Efficacy is easiest to evaluate when (1) the practice fully determines the outcome, (2) the practice's action is observable, and (3) the outcome occurs immediately after use. ${ }^{32}$ Most familiar technologies exhibit these features. Swinging a hammer fully determines whether a nail goes into a piece of wood, the hammer's action is observable, and the nail is forced inwards immediately after the hammer is swung. Similarly, striking a match fully determines whether a flame appears, the match's action is observable, and the flame appears as soon as the match is struck.

In contrast, practices whose efficacy is hard to evaluate seem much less likely to develop. Researchers have noted the incredible benefits that come from sanitation practices like handwashing, boiling water, and building latrines. ${ }^{71-74}$ Yet despite their demonstrated benefits, 
such practices diffuse slowly and didn't seem to spontaneously culturally evolve in many communities. ${ }^{74,75}$ The likely reason seems to be the difficulty of evaluation. Individuals cannot easily gauge the benefits of sanitation practices because of the long temporal lag, as well as the many other factors determining health, making it difficult to pinpoint any single practice's contribution. Notably, these practices arose and spread only after people developed new technologies of evaluating usefulness (e.g., rigorous experimentation), a point to which I'll later return.

\section{Explaining the evolution of aesthetic traditions}

Why do people tell stories, and why do they exhibit the features they do? Why do they create art or sing love songs? Many researchers argue that these behaviors develop, either through genetic or cultural evolution, because they promote individual- or group-level benefits. ${ }^{76,77}$ But according to the account developed here, aesthetic traditions such as these might develop through subjective selection as people craft them to satisfy regular goals.

Perhaps the most important goal of aesthetic culture is entertainment. Audiences like to experience pleasure. Producers (e.g., storytellers or artists) benefit from inducing it. ${ }^{12}$ As audiences demand their favorites and producers choose variants that previously proved effective, they drive a selection for pleasure-inducing culture. Ethnographers have observed this process in action. In his study of ntsomi story-performers among the Xhosa of South Africa, Scheub wrote that "an artist includes and emphasizes those elements that she delighted in during $n t s o m i$ performances that she witnessed, and she does not fail to recall those details that particularly delighted her audiences during her own productions"78 (p. 90). He even connected this subjective selection to the evolution of the ntsomi tradition: "Considering that this process of borrowing, influencing, innovating, and combining has been going for decades, there should be no surprise that such an involved form has developed"78 (p. 19).

Not only does the impulse to entertain (and be entertained) drive the evolution of many aesthetic traditions, but it also explains profound cross-cultural patterns. Evolutionary and cognitive researchers have noted how recurrent features of stories, from coalitional conflict to the triumph of the heroic orphan, seem well designed to draw attention and evoke pleasure. ${ }^{79,80}$ The same goes for widespread features of visual art, such as circles, straight lines, and horizontal symmetry, ${ }^{81}$ and even the pleasing elements of manufactured landscapes ${ }^{82}$ (e.g., grassy greens and watery blues). ${ }^{83}$ Universals in aesthetic traditions may reflect the capacity for subjective selection to craft culture that people want to consume.

Of course, entertainment is just one of many goals that people pursue when sharing and consuming aesthetic traditions. Music, for example, is consistently used for such diverse ends as dancing, soothing infants, healing illness, communicating love, greeting visitors, and accompanying processions. ${ }^{84,85}$ Moreover, songs that share behavioral functions tend to exhibit common musical features. ${ }^{84,85}$ These form-function relationships may be the result of subjective selection: As people search for the best ways to heal illness or calm infants or greet visitors, they may craft musical practices compellingly designed to achieve those goals.

\section{Explaining the evolution of cooperative and oppressive institutions}


The subjective selection framework also provides an account for the evolution of institutions, one that builds on recent research on the roles of self-interest and foresight in shaping rules. ${ }^{86-89}$ Just as people selectively retain tools and aesthetic practices that appear to achieve their aims, they do the same for institutions: They build, enforce, and maintain sets of rules that satisfy psychologically-determined ends - typically their (perceived) material self-interest. ${ }^{89}$

More than the other cultural domains discussed, the cultural evolution of rules and institutions often involves conflicts of interest, and outcomes depend on the relative bargaining power and the degree of overlapping interests among parties. When decision-makers' goals overlap with the long-term interests of their groups, they assemble systems of group-functional rules. ${ }^{89}$ Ostrom, for example, famously documented cases of communities developing small-scale institutions to manage common-pool resources. ${ }^{90}$ One of those cases involved fishermen in Alanya, Turkey, who recognized the costs that came from overharvesting a shared fishery. Determined to develop a more sustainable scheme, they experimented with different arrangements over a 15-year-period, iteratively retaining effective rules and, in turn, producing a successful system that sustained cooperation. ${ }^{91}$ Similarly, pirates of the $17^{\text {th }}$ and $18^{\text {th }}$ centuries crafted constitutions to regulate behavior aboard their ships. Importantly, pirates agreeing to a new constitution selectively adopted rules from previous constitutions, evaluating them and tweaking them into more effective forms. ${ }^{92,93}$ Fox $^{92}$ observed that some pirate captain's codes, like that of Bartholomew Roberts (Black Bart), were a combination of effective rules those captains encountered and new rules that satisfied the captains' own idiosyncratic preferences. Notably, these cases are examples of subjective selection, because people adopt and retain practices evaluated as satisfying their goals, as well as what scholars call cultural group selection, ${ }^{94}$ or the process in which cultural variants that promote group-level success spread at the expense of less group-beneficial variants.

The outcomes of institutional tugs-of-war differ when there are stark asymmetries in power and decision-makers' interests diverge from other group members. Decision-makers still craft and adopt rules evaluated as serving their interest, but often at the expense of other group members. An example is the evolution of laws enforcing racial segregation in the American South. Known as Jim Crow, these laws developed as white legislators devised schemes to disenfranchise blacks, adopting oppressive arrangements from neighboring states. As Woodward wrote, "states elaborated the original scheme and added devices of their own contriving... there was a great deal of borrowing and interchange of ideas throughout the South" (p. 83). ${ }^{95}$ Groups produce institutions that promote cooperation, yes, but they also develop rules that are oppressive or predatory. A consideration of enforcers' goals helps explain why.

\section{Cultural dependencies}

Cultural variants often seem intertwined. A technology emerges and industries grow around it. A new belief enters a community, and a new suite of practices seem to follow. An aim of the naturalistic study of culture is to understand these interlocking relationships- to understand, in a sense, the ecology of culture.

The framework presented here identifies at least two broad mechanisms by which cultural variants might affect each other. First, a variant can affect how people evaluate new practices or beliefs. For instance, a variant might change people's causal model of the environment, transforming how they evaluate other variants. Second, a variant can create new goals, in turn 
creating new niches for practices to fill. For instance, a technology might create new problems, demanding new solutions. As I will show, both seem to occur.

\section{Cultural variants affect the mental causal models in which new variants are evaluated}

According to this framework, culture evolves as people evaluate which variants best satisfy their goals. By that logic, if a culturally transmitted variant affects people's evaluation criteria, then other cultural practices should, in turn, be affected.

One way that culture affects people's evaluation criteria is through their beliefs. I reviewed how behaviors are often evaluated in causal models of the environment. Because these models are built from culturally transmitted beliefs, people's evaluation criteria will be affected by people's cultural environments. This is clear when considering how people's mental models of uncertainty determine the specialists they visit. Various writers have compared shamans to money managers, because both are magicians of uncertainty: Both use opaque methods to convince clients they can control desired but ultimately unpredictable outcomes. ${ }^{96,97}$ Yet a critical difference between the two is the causal model of uncertainty they work with. Shamans exist in contexts where the dominant model is that uncertain outcomes are caused by invisible agents. To attract clients, they must therefore engage with those agents, such as by entering trance and communing with spirits. ${ }^{64}$ Money managers, meanwhile, adapt to different, prevailing understandings, such as that the market, while unpredictable, functions according to complex trends. They must, in turn, convince clients that they can divine these trends, such as by using convoluted mathematical models. People's preferences for specialists hinge on culturallytransmitted models of uncertainty, in turn shaping the services specialists provide.

A more dramatic example of how culturally transmitted variants alter evaluation are the effects of the scientific revolution. Wootton compared the beliefs of a hypothetical, welleducated Englishman before and after the scientific revolution. ${ }^{98}$ The Englishman before the scientific revolution - say, in 1600-believed in witches, werewolves, alchemy, astrology, unicorns, sympathetic magic, the factual nature of the Odyssey, and the spontaneous generation of mice. His post-revolution counterpart-say, in 1733-believed in none of these. The difference? Their criteria for evaluating information and practices. Between 1600 and the early 1700s, educated Europeans developed an epistemological toolkit that centered on direct experience and rigorous experimentation as grounds for evaluating information and technology. ${ }^{98}$ The particular innovations or events that drove this transition are beyond the scope of this paper. Nevertheless, the scientific revolution demonstrates that culturally transmitted variants can affect our evaluation criteria and, as a result, other cultural variants. In fact, the monumental effects of the scientific revolution, including upheavals of both beliefs and technology ${ }^{98}$, demonstrate the importance of subjective evaluation in determining the form of culture. Overhaul how people evaluate culture, and culture transforms.

\section{Cultural variants create new goals}

Technologies and beliefs can also create new goals, altering the selective landscape for cultural variants. This might occur because culturally transmitted beliefs change people's understanding of the world, making them prioritize goals that were previously unimportant. For example, once people accept that illness is caused by witchcraft (a culturally transmitted belief), they are 
motivated to pursue new goals stemming from that belief, such as protecting themselves, identifying who attacked them, and healing illness caused by evil magic. These goals, in turn, generate a litany of corresponding magical technology, including protective magic, divination, and witch doctoring. The ubiquitous co-occurrence of witchcraft beliefs and technology for fighting witchcraft illustrates how culturally transmitted beliefs open up corresponding technological niches (see Box 2). ${ }^{99}$

Just as new beliefs produce goals, new technology does, as well. This can occur for one of three reasons. ${ }^{100}$ First, technology can provide people with new information, which, in combination with previous goals, produces new sub-goals. For instance, people are motivated to be healthy. If a technology reveals that a particular factor (like diabetes) contributes to negative health outcomes, people will be motivated to control that harmful factor, opening up a new cultural niche. Second, the production, maintenance, and dissemination of a technology can become a new goal. As automobiles grew in popularity, a new set of needs centered on distributing and repairing cars followed. Finally, technologies can create problems that people must address. These problems can take many forms: A new military technology might create a need to defend against it; an extractive technology might create new environmental hazards that threaten people's welfare. These needs create new niches for novel technologies to fill. Technology is a mother of necessity.

\section{Box 2. Witchcraft as a case study of cultural dependencies.}

To appreciate how subjective selection might explain how different cultural variants affect each other, consider the beliefs and practices surrounding witchcraft. In his monograph Witchcraft, Oracles, and Magic Among the Azande, Evans-Pritchard famously showed that, to explain many features of $Z$ ande symbolic culture, one had to explain the web of practices and beliefs within they existed. ${ }^{101}$ The book's cover, displayed as panel A below, illustrated these relationships by linking three features of Zande culture-mangu (witchcraft), soroka (oracles), and ngua (magic or medicines)— together in a triangle.

Panel B presents a proposal for the origins of these cultural dependencies. The desire to harm others (a goal) fuels a cultural selection for effective-seeming harmful magic (a practice). ${ }^{102}$ Once people suspect that others use harmful magic, it becomes a compelling explanation of illness (a belief). The belief in turn, produces a suite of new goals rooted in combatting it, such as deterring bad magic, figuring out who performed it, and healing illnesses caused by it. As people choose and pass on the most effective-seeming means of satisfying these goals, they drive the evolution of new forms of attractive magic (practices). By affecting people's goals and beliefs, a single culturally evolved practice can eventually give rise to a cultural ecosystem. 


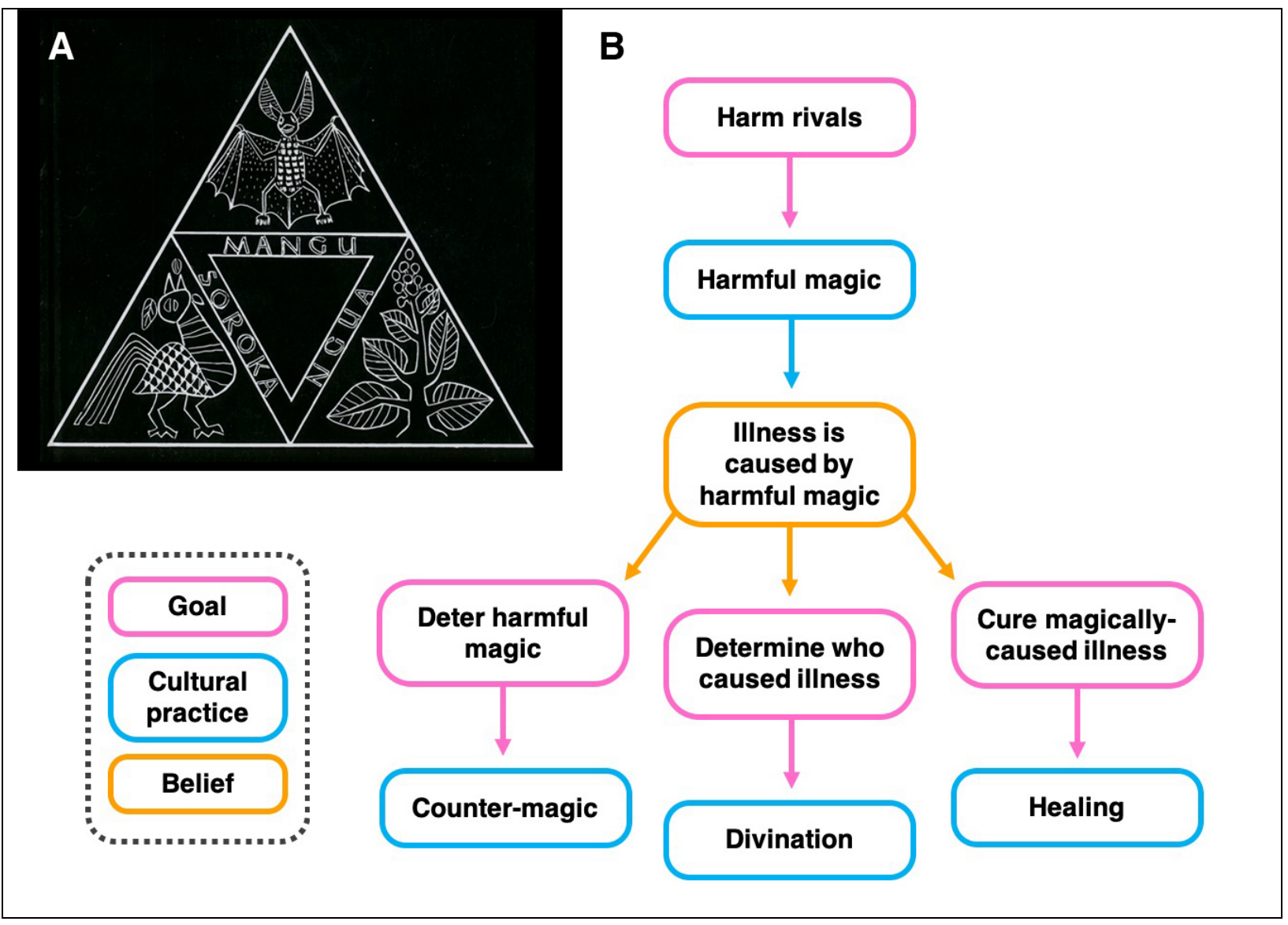

Interactions with other proposed cultural evolutionary processes

Subjective selection is broadly consistent with other cultural evolutionary frameworks. From the perspective of cultural attraction, subjective selection describes how people's goals and the criteria for evaluating them together produce attractors in the space of possible cultural configurations. Unlike so-called "narrow" cultural attraction (see ref. ${ }^{103}$ ), however, subjective selection posits that cultural design emerges from both goal-directed production and selection, although it is consistent with the idea that features of human psychology constrain how people reconstruct culture during transmission. From the perspective of dual inheritance theory, subjective selection reflects a combination of 'guided variation', as people craft goal-directed culture, and a 'content bias' during cultural transmission which favors practices evaluated as satisfying people's goals.

Despite this overall consistency, and as stated at the outset, the roles of goals and evaluation have remained surprisingly undeveloped in cultural evolutionary theory. ${ }^{104}$ As a result, subjective selection differs in important ways from many of the cultural evolutionary processes that have been hypothesized and used to explain culture.

\section{Rational actor models}

Subjective selection shares features with rational actor models of culture: Individuals produce and preferentially retain culture that satisfies self-interested goals. ${ }^{90,105}$ However, the focus on 
subjective appeal gives the subjective selection framework a psychological richness, and thus an explanatory power, lacking in many rational actor approaches to culture. ${ }^{105}$ Instead of assuming simply that people pursue self-interested ends, subjective selection sees people's goals either as outcomes that were correlated with higher fitness during our evolutionary history (e.g., food, revenge, information) or as the hierarchy of subordinate goals that have been learned to achieve these adaptive outcomes (e.g., trek out to forest, shoot monkey, butcher meat). In this way, the subjective selection framework is similar to earlier approaches by Alexander ${ }^{106,107}$, who enriched the rational actor model with an evolutionary approach to human motivation. Subjective selection further differs from many rational actor approaches, including Alexander's, by focusing on the psychology of evaluation. Our cognitive architecture systematically biases how we evaluate cultural practices, favoring cultural traditions that are hard to explain under many rational actor models.

To appreciate the added explanatory power of incorporating psychology, consider the many inefficiencies reviewed earlier. People create suboptimal baseball teams. They visit shamans and money managers. Latrines, handwashing, and water-boiling practices diffuse slowly, if at all. Such inefficiencies cannot be explained without considering the psychological processes involved in evaluation. Or, similarly, consider beliefs in witches. According to one subjective selection hypothesis, such beliefs may develop because people are motivated to adopt explanations of misfortune and, under conditions of uncertainty and persecution, they evaluate explanations blaming distrusted individuals as more likely. ${ }^{102}$ This hypothesis, which considers both an adaptive, goal-directed motivation (adopting explanations of misfortune) and biased evaluation criteria (favoring conspiratorial explanations in circumstances of distrust), explains many design features of beliefs in witches, such as their personality traits, the types of misfortunes they cause, and the animals with which they associate (reviewed in ref. ${ }^{102}$ ). A rational actor model, in contrast, has difficulty accounting for these beliefs and their properties.

\section{Traditional cultural attraction}

I use 'traditional cultural attraction' to refer to any cultural evolutionary process in which particular regions of the potential cultural design-space are favored during cultural transmission because they are (1) especially attention-grabbing or arousing (such as portraits that stare at the subject ${ }^{108}$; (2) especially memorable (such as religious representations that minimally violate folk-ontological categories) ${ }^{109}$; or (3) more likely to be reconstructed (such as folk biological taxonomies ${ }^{110}$. I use the restrictive term 'traditional cultural attraction' for two reasons. First, the umbrella term 'cultural attraction' has been used variably in the cultural evolutionary literature, sometimes apparently encompassing all directional cultural change (see, for example, the discussion in ref. ${ }^{103}$ ). Second, as I wrote above, subjective selection seems broadly consistent with most versions of cultural attraction theory with the exception of its emphasis on biased production and selection. 'Traditional cultural attraction' thus refers to the distinct set of hypothesized processes on which cultural attraction theorists have focused.

The major difference between subjective selection and traditional cultural attraction is the role of goal-directed behavior (although see refs. ${ }^{111,112}$ ). To explain culture, traditional cultural attraction theorists have focused on how variants resemble the inputs of cognitive mechanisms or the inferences those mechanisms generate. ${ }^{13}$ For instance, because of cognitive mechanisms for tracking and responding to faces, humans seem to find masks or particular portraits appealing or 
arousing. ${ }^{108,113}$ Or, because of cognitive mechanisms for thinking about agents, humans seem to reconstruct beliefs about gods in patterned ways. ${ }^{114}$ Subjective selection, meanwhile, prioritizes people's instrumental goals and the criteria they have use for evaluating whether those goals have been achieved.

This difference in focus means that subjective selection can explain many behaviors that traditional cultural attraction cannot. Perhaps most important is the appearance of function. Aesthetics, ritual, and symbolic culture are not just cognitively compelling; they seem functional. Stories seem designed to entertain or socialize. Magical practices seem designed to acquire opaque information or control uncertain outcomes. Explaining functional design requires considering people's instrumental reasons for producing or consuming a cultural variant and how those instrumental reasons determine the variant's features. The inability of traditional cultural attraction to explain functionality is clearest when considering useful technologies. Whereas subjective selection easily explains these as developing from iterated goal-directed evaluation and retention, traditional cultural attraction has difficulty explaining useful technology. In fact, useful technology is often said to be outside of the explanatory jurisdiction of traditional cultural attraction..$^{9-11}$

As a result of these differences, subjective selection also provides alternative explanations for aspects of culture often explained using traditional cultural attraction. Take beliefs in supernatural beings. Boyer argued that such beliefs persist, in part, because they are memorable. ${ }^{109}$ By his account, humans have ontological categories. When an entity is identified as belonging to one of those categories, such as 'person', specific inferences are triggered, such as 'person cannot walk through walls'. Representations that minimally violate these inferences, like of a person who can walk through walls, are more memorable and thus more likely to be encoded and communicated. In contrast, a subjective selection account might hypothesize that people are motivated to search for information under particular circumstances and that beliefs in invisible agents satisfy the intuitive evaluation criteria for adopting that information. ${ }^{115}$ For example, people may be motivated to search for explanations of serendipitous events, and supernatural agents may best satisfy the psychological criteria for a plausible explanation.

Still, subjective selection hypotheses are not necessarily mutually exclusive with traditional cultural attraction accounts. Rather, subjective selection might explain some features of a cultural product while traditional cultural attraction explains others. It might be the case that people believe in invisible agents because they are motivated to search for explanations. Yet of those variants that satisfy evaluation criteria, some may also be especially memorable or attention-grabbing. In this case, subjective selection would explain why people adopt beliefs in spirits and why those beliefs exhibit some traits, while the enhanced transmission of memorable variants would explain other features.

\section{Other cultural evolutionary processes}

Some cultural evolutionary researchers argue that individual- or group-adaptive culture can evolve without individuals evaluating them for their particular uses. In such cases, the objective benefits of a variant - its effect on individual health or group-level competitiveness, for example-determines its subsequent success. Researchers have proposed several mechanisms by which such adaptive cultural evolution might occur: ${ }^{4,6,45}$ 
1. Cultural evolution by natural selection. Assuming that culture is faithfully transmitted from parents to children, practices that promote dimensions of fitness (e.g., health, strength, fertility) should spread through a population as their bearers proliferate.

2. Adaptive cultural evolution through cue-biased transmission. People preferentially learn from parties who exhibit cues of useful cultural knowledge, such as health, success, strength, and prestige. As a consequence, cultural practices that maximize metrics like health, strength, and prestige will spread at the expense of others.

3. Cultural group selection. As groups compete, groups with group-beneficial cultural variants outcompete those with less competitive culture, driving the spread of group-adaptive variants.

Researchers often consider how these processes can interact with regular features of the human mind to shape culture. ${ }^{116}$ Henrich and Henrich, for instance, studied how both cognitive responses to categorical anomalies and cultural evolution driven by cue-biased transmission might explain features of Fijian food taboos ${ }^{117}$. Norenzayan et al., meanwhile, considered how cognitive biases constrain religious representations with cultural group selection subsequently selecting for those representations most potent at promoting group-level benefits.

An important implication of these hypotheses is that these processes can produce adaptive design without individuals evaluating (or even understanding) the value of variants. Through competition, differential reproduction, and biased transmission, people adopt practices that make them and their groups healthier, stronger, and more competitive while remaining ignorant of how those practices advantage them.

How do subjective selection and these other cultural evolutionary hypotheses interact? For one, subjective selection provides alternative explanations. For example, many researchers assume or argue that magic, divination, witchcraft beliefs, and shamanism develop to provide individual- or group-level benefits, including social bonding, enforcing cooperation, and promoting confidence (see reviews in refs. ${ }^{102,118}$ ). But as demonstrated, objective benefits are not necessary to explain these traditions; rather, they can survive and spread simply by satisfying people's evaluation criteria for achieving regular goals. ${ }^{64,102}$ Similarly, complex, useful technologies may evolve through the three processes just listed. But it also might be the case that they develop through iterated trial-and-error, as people evaluate how well practices seem to satisfy a goal, retaining what looks to work and iteratively building more effective-seeming culture.

Again, however, these processes are not necessary mutually exclusive: Subjective selection might interact with objective selection processes, such as cultural group selection, to jointly shape traditions. Take, for example, institutions of punitive justice, such as the makarrata ceremony of northern Australia in which an injured party stabbed the thigh of an aggressor. ${ }^{119}$ Such institutions may develop simply to satisfy the joint goals of revenge and reinstating cooperation: Injured parties want retribution and to restore goodwill, and they and their aggressors devise and retain those methods that best achieve these goals. But it might also be the case that cultural group selection subsequently selects among institutions of punitive justice, driving the spread of group-functional variants.

\section{Applying subjective selection and testing novel predictions}


The framework developed here is widely applicable. It generates hypotheses for any practice or belief, and those hypotheses yield testable predictions:

Say we hypothesize that a cultural item evolves as people iteratively evaluate and

selectively retain variants that appear to best satisfy a given goal. We would predict the following:

1. People pursuing that goal will adopt the item more than people not pursuing the goal. In experimental settings, goals might be manipulated with explicit instruction or by coupling an outcome with a monetary reward.

2. Providing people with an item that appears to better satisfy the goal (measured, for instance, with ratings of apparent efficacy and ease of use) will reduce their reliance on the item of interest.

3. In experimental settings, manipulating the apparent efficacy of the item for satisfying the goal will affect people's reliance on it, even when controlling for total reward.

4. In experimental settings, manipulating the apparent efficacy of the item for satisfying the goal, especially in unexpected ways, will elicit activity in brain regions that encode reward prediction error for goal-directed activities (see, e.g., ref. ${ }^{23}$ ). Activity will occur in regions associated with negative prediction error when the item is made to seem less effective and in regions associated with positive prediction error when the item seems more effective.

5. In experimental settings, manipulating the apparent efficacy of the item for satisfying the goal will have a larger effect on participants' use of the item when pursuing the same goal compared to when pursuing other goals.

For example, if we hypothesize that people develop a ritual to change the weather, we should expect: (1) individuals will use the ritual more when they have the goal of changing the weather (as elicited, say, by instruction or monetary reward); (2) individuals will use the ritual less if they encounter a more effective-seeming method of changing the weather (as measured, say, by ratings of efficacy and ease of use); (3) individuals will use the ritual more if it is experimentally (and subjectively) made to seem more effective at changing the weather (again, potentially measured by subjective ratings of efficacy and ease of use); (4) there will be activity in brain structures that encode positive reward prediction error following an abrupt increase in the frequency of apparent successes in changing the weather; and (5) individuals will use the ritual more for changing the weather than for other goals following a string of apparent successes in changing the weather. In the preceding sentences, the words ritual and changing the weather can be replaced with any other pairing of a cultural item and a goal, such as tool and bunting an animal, ceremony and restoring cooperation, institution and protecting a public good, song and healing a patient, and so on.

The above predictions avoid what might seem like a circularity in the account. Although any cultural item can be stipulated to have developed because it apparently satisfies any goal, each item-goal pairing is testable. If one hypothesizes that a ritual culturally evolved and is stable because it apparently satisfies the goal of inviting rain, yet then none of the above predictions holds, then we would reject that hypothesis.

Beyond testing a particular item-goal pairing, we can also test whether a cultural item exhibits certain features, because, given a particular causal model, people evaluate that item as effective. Such a hypothesis yields the following predictions:

6. Individuals with the relevant causal model will be more likely to adopt the item than individuals who do not subscribe to that model. 
7. Changing people's causal models will affect their use of the cultural item-and especially of variants that exhibit the relevant features.

8. Changing the features of interest will have a greater effect on the likelihood of using the item among people who have the relevant causal model than among people who do not. For example, if we hypothesize that a weather ritual involves communing with spirits because people believe that spirits oversee rain, we should expect: (1) individuals who believe that spirits cause rain ("believers") will be more likely to adopt the ritual than individuals who do not believe ("non-believers"); (2) disruptions to beliefs about the causes of rain will affect the likelihood with which people use the weather ritual, especially those versions of the ritual that involve spirit communion; and (3) there will be a greater change to ritual use among believers compared to non-believers after the spirit communion has been removed. Again, beyond discussing weather rituals, spirit communion, and beliefs that spirits cause rain, we can apply these predictions to any cultural variant whose success, we hypothesize, is attributable to the causal model in which it is evaluated.

Finally, the framework developed here makes predictions about the cumulative cultural evolution of technology, whether effective or ineffective:

9. A given cultural solution will be less likely to cumulatively evolve when individuals in a transmission chain do not share goals—or even when they cannot infer each other's goals.

10. Elaborate, costly, and ineffective practices can develop and be sustained over many generations as long as people regard them as effective for their regular goals, regardless of whether those solutions provide compensatory benefits.

\section{Culture as cheesecake}

Steven Pinker famously referred to music as "auditory cheesecake"- "an exquisite confection crafted to tickle the sensitive spots of at least six of our mental faculties"83 (p. 534). Music, he argued, didn't develop because it provides adaptive benefits. Rather, it exists because it delivers exactly what human minds want. As cultural evolution and ingenious tastemakers selectively pass on variants that people desire most, they fashion music into complex, highly appealing forms.

An implication of the subjective selection framework is that the cheesecake analogy applies not just to music but to major swaths of culture. People constantly assemble and maintain practices and beliefs to satisfy their goals, regardless of whether those cultural items provide actual material benefits. Whether we consider chairs, brownies, hammers, igloos, rain magic, shamanism, bows-and-arrows, or institutions that protect against the overexploitation of resources, cultural products of all kinds develop as people preferentially adopt culture that seems to give them what they want. 


\section{References}

1 Pulliam HR, Dunford C. 1980. Programmed to learn: An essay on the evolution of culture. Columbia University Press.

2 Boehm C. 1978. Rational Preselection from Hamadryas to Homo Sapiens: The Place of Decisions in Adaptive Process. Am Anthropol 80:265-296.

3 Ruyle EE. 1973. Genetic and cultural pools: Some suggestions for a unified theory of biocultural evolution. Hum Ecol 1:201-215.

4 Boyd R, Richerson PJ. 1985. Culture and the evolutionary process. Chicago: University of Chicago Press.

5 Henrich J, McElreath R. 2008. Dual inheritance theory: The evolution of human cultural capacities and cultural evolution. In: Dunbar R, Barrett L, editors. Oxford Handb. Evol. Psychol. Oxford, UK: Oxford University Press. p 555-570.

6 Henrich J. 2016. The secret of our success: How culture is driving human evolution, domesticating our species, and making us smarter. Princeton, NJ: Princeton University Press. 7 Morin O. 2016. How traditions live and die. New York: Oxford University Press.

8 Heath $\mathrm{C}$ et al. 2001. Emotional selection in memes: The case of urban legends. J Personal Soc Psycology 81:1028-1041.

9 Sterelny K. 2017. Cultural evolution in California and Paris. Stud Hist Philos Biol Biomed Sci Elsevier Ltd. 62:42-50.

10 Scott-Phillips T et al. 2018. Four misunderstandings about cultural attraction. Evol Anthropol 27:162-173.

11 Richerson PJ. 2017. Recent critiques of dual inheritance theory. Evol Stud Imaginative Cult 1:203-212.

12 André J-B et al. 2020. The Mystery of Symbolic Culture: What fitness costs? What fitness benefits? OSF Prepr :1-18.

13 Custers R, Aarts H. 2010. The unconscious will: How the pursuit of goals operates outside of conscious awareness. Science (80- ) 329:47-50.

14 Kruglanski AW et al. 2015. The rocky road from attitudes to behaviors: Charting the goal systemic course of actions. Psychol Rev 122.

15 Botvinick MM et al. 2009. Hierarchically organized behavior and its neural foundations: A reinforcement learning perspective. Cognition 113:262-280.

16 Botvinick MM. 2012. Hierarchical reinforcement learning and decision making. Curr Opin Neurobiol Elsevier Ltd. 22:956-962.

17 Barrett HC. 2015. The shape of thought: How mental adaptations evolve. New York: Oxford University Press.

18 Gershman SJ. 2015. Reinforcement learning and causal models. In: Waldmann M, editor. Oxford Handb. Causal Reason. Oxford University Press. p 295-306.

$19 \mathrm{Kool} \mathrm{W}$ et al. 2018. Competition and cooperation between multiple reinforcement learning systems. Goal-Directed Decis. Mak. Comput. Neural Circuits.

20 Keramati M et al. 2011. Speed/accuracy trade-off between the habitual and the goal-directed processes. PLoS Comput Biol 7:e1002055.

21 Cushman F, Morris A. 2015. Habitual control of goal selection in humans. Proc Natl Acad Sci 112:13817-13822.

22 O’Doherty JP et al. 2017. Learning, Reward, and Decision Making. Annu Rev Psychol 
68:73-100.

23 Ribas-Fernandes JJF et al. 2011. A neural signature of hierarchical reinforcement learning. Neuron 71:370-379.

24 Diuk C et al. 2013. Hierarchical learning induces two simultaneous, but separable, prediction errors in human basal ganglia. J Neurosci 33:5797-5805.

25 Laland K, Evans C. 2017. Animal social learning, culture, and tradition. APA Handb. Comp. Psychol. p 441-460.

26 Worden BD, Papaj DR. 2005. Flower choice copying in bumblebees. Biol Lett 1:504-507. 27 Danchin É et al. 2019. Cultural flies: Conformist social learning in fruitflies predicts longlasting mate-choice traditions. Science (80- ) 362:1025-1030.

28 Whiten A. 2017. Social learning and culture in child and chimpanzee. Annu Rev Psychol 68:129-154.

29 Glowacki L, Molleman L. 2017. Subsistence styles shape human social learning strategies. Nat Hum Behav 1:0098.

30 Toelch U et al. 2013. Individual consistency and flexibility in human social information use. Proc R Soc B Biol Sci 281.

31 Morgan TJH et al. 2012. The evolutionary basis of human social learning. Proc R Soc Ser B, Biol Sci 279:653-662.

32 Rogers E. 2003. The diffusion of innovations. New York: The Free Press.

33 Enquist $M$ et al. 2007. Critical Social Learning: A Solution to Rogers's Paradox of Nonadaptive Culture. Am Anthropol 109:727-734.

34 Aoki K. 2010. Evolution of the social-learner-explorer strategy in an environmentally heterogeneous two-island model. Evolution (N Y) 64:2575-2586.

35 Lehmann L et al. 2013. On optimal learning schedules and the marginal value of cumulative cultural evolution. Evolution (N Y) 67:1435-1445.

36 Heyes C. 2012. What's social about social learning? J Comp Psychol 126:193-202.

37 Olsson A et al. 2020. The neural and computational systems of social learning. Nat Rev Neurosci Springer US. 21:197-212.

38 Csibra G, Gergely G. 2007. "Obsessed with goals”: Functions and mechanisms of teleological interpretation of actions in humans. Acta Psychol (Amst) 124:60-78.

39 Wood LA et al. 2013. Whom do children copy? Model-based biases in social learning. Dev Rev Elsevier Inc. 33:341-356.

40 Carpenter $\mathrm{M}$ et al. 2005. Twelve- and 18-month-olds copy actions in terms of goals. Dev Sci 8:13-20.

41 Lyons DE et al. 2007. The hidden structure of overimitation. Proc Natl Acad Sci U S A 104:19751-19756.

42 Hoehl S et al. 2019. 'Over-imitation': A review and appraisal of a decade of research. Dev Rev Elsevier. 51:90-108.

43 Kline MA et al. 2020. Irrelevant-action imitation is short-term and contextual: Evidence from two under-studied populations. Dev Sci 23:1-15.

44 Mercier H. 2020. Not born yesterday: The science of who we trust and what we believe. Princeton University Press.

45 Durham WH. 1991. Coevolution: Genes, culture, and human diversity. Stanford University Press.

46 Richerson PJ, Boyd R. 2008. Not by genes alone: How culture transformed human evolution. 
Chicago: University of Chicago Press.

47 Derex M, Mesoudi A. 2020. Cumulative cultural evolution within evolving population structures. Trends Cogn Sci Elsevier Ltd. 24:654-667.

48 Chua RYJ et al. 2015. The Impact of Culture on Creativity: How Cultural Tightness and Cultural Distance Affect Global Innovation Crowdsourcing Work. Adm Sci Q 60:189-227. 49 Sherman PW, Billing J. 1999. Darwinian Gastronomy: Spices taste good because they are good for us. Bioscience 49:453-463.

50 Allen KR et al. 2020. Rapid trial-and-error learning with simulation supports flexible tool use and physical reasoning. Proc Natl Acad Sci 117:29302-29310.

51 Mccarthy W et al. 2020. Learning to build physical structures better over time. Proc. 42nd Annu. Meet. Cogn. Sci. Soc.

52 Ullman TD et al. 2017. Mind Games: Game Engines as an Architecture for Intuitive Physics. Trends Cogn Sci Elsevier Ltd. 21:649-665.

53 Miton H, Charbonneau M. 2018. Cumulative culture in the laboratory: Methodological and theoretical challenges. Proc R Soc B 285:20180677.

54 Zwirner E, Thornton A. 2015. Cognitive requirements of cumulative culture: Teaching is useful but not essential. Sci Rep Nature Publishing Group. 5:16781.

55 Caldwell CA et al. 2016. Experimental approaches to studying cumulative cultural evolution. Curr Dir Psychol Sci 25:191-195.

56 Derex M et al. 2019. Causal understanding is not necessary for the improvement of culturally evolving technology. Nat Hum Behav Springer US. 3:446-452.

57 Derex $M$ et al. 2018. Divide and conquer: Intermediate levels of population fragmentation maximize cultural accumulation. Philos Trans R Soc B Biol Sci 373.

58 Thaler R, Sunstein C. 2004. Market efficiency and rationality: The peculiar case of baseball. Mich Law Rev 102:1390-1403.

59 Padoa-Schioppa C. 2011. Neurobiology of Economic Choice: A Good-Based Model. Annu Rev Neurosci 34:333-359.

60 Beck J, Forstmeier W. 2007. Superstition and belief as inevitable by-products of an adaptive learning strategy. Hum Nat 18:35-46.

61 Damisch L et al. 2010. Keep your fingers crossed! How superstition improves performance. Psychol Sci 21:1014-1020.

62 Moore OK. 1957. Divination-A new perspective. Am Anthropol 59:69-74.

63 Glowacki L. 2018. The cultural evolution of war rituals. Behav Brain Sci 41:e74.

64 Singh M. 2018. The cultural evolution of shamanism. Behav Brain Sci 41:e66.

65 Winkelman MJ, White D. 1987. A cross-cultural study of magico-religious practitioners and trance states: Database. HRAF Res Ser Quant Cross-Cultural Data III.

66 Peoples HC et al. 2016. Hunter-gatherers and the origins of religion. Hum Nat Human

Nature.

67 Vyse S. 2014. Believing in magic: The psychology of superstition. Oxford and New York: Oxford University Press.

68 Winkelman M. 2000. Shamanism: The neural ecology of consciousness and healing.

Westport, CT: Bergin \& Garvey.

69 Singh M, Henrich J. 2020. Why do religious leaders observe costly prohibitions? Examining taboos on Mentawai shamans. Evol Hum Sci 2:e32.

70 Swanson GE. 1964. The birth of the gods: The origin of primitive beliefs. Ann Arbor: The 
University of Michigan Press.

71 Dinkel KA et al. 2020. Relationship of sanitation, water boiling, and mosquito nets to health biomarkers in a rural subsistence population. Am J Hum Biol 32:1-13.

72 Cohen A, Colford JM. 2017. Effects of boiling drinking water on diarrhea and pathogenspecific infections in low- and middle-income countries: A systematic review and meta-analysis. Am J Trop Med Hyg 97:1362-1377.

73 Bloomfield SF, Nath KJ. 2009. Use of ash and mud for handwashing in low income communities: An IFH expert review. Home Hyg Heal.

$74 \mathrm{Fewtrell} \mathrm{L}$ et al. 2005. Water, sanitation, and hygiene interventions to reduce diarrhoea in less developed countries: A systematic review and meta-analysis. Lancet Infect Dis 5:42-52.

75 Pengpid S, Peltzer K. 2012. Hygiene behaviour and health attitudes in African countries. Curr Opin Psychiatry 25:149-154.

76 Smith D et al. 2017. Cooperation and the evolution of hunter-gatherer storytelling. Nat Commun Springer US. 8.

77 Dissanayake E. 2008. The arts after Darwin: Does art have an origin and adaptive function? In: Zijlmans K, van Damme W, editors. World Art Stud. Explor. Concepts Approaches. Amsterdam: Valiz. p 241-263.

78 Scheub H. 1975. The Xhosa ntsomi. Oxford: Clarendon Press.

79 Nettle D. 2005. The wheel of fire and the mating game: Explaining the origins of tragedy and comedy. J Cult Evol Psychol 3:39-56.

80 Singh M. 2021. The sympathetic plot, its psychological origins, and implications for the evolution of fiction. Emot Rev 13:183-198.

81 Boas F. 1955. Primitive art. New York: Dover Publications, Inc.

82 Moura JMB et al. 2017. Landscape preferences in the human species: Insights for ethnobiology from evolutionary psychology. Ethnobiol Conserv 6:1-8.

83 Pinker S. 1997. How the mind works. New York and London: W. W. Norton \& Company. $84 \mathrm{Mehr}$ SA et al. 2018. Form and function in human song. Curr Biol 28:356-368.

85 Mehr SA et al. 2019. Universality and diversity in human song. Science (80- ) 366:eaax0868. 86 Powers ST. 2018. The institutional approach for modeling the evoluton of human societies. Artif Life 24:10-28.

87 Perry L et al. 2018. Collective Action Problem in Heterogeneous Groups with Punishment and Foresight. J Stat Phys 172:293-312.

88 Gavrilets S, Shrestha MD. 2020. Evolving institutions for collective action by selective imitation and self-interested design. Evol Hum Behav Elsevier Inc.

89 Singh $M$ et al. 2017. Self-interest and the design of rules. Hum Nat 28:457-480.

90 Ostrom E. 1990. Governing the commons: The evolution of institutions for collective action. Cambridge, UK: Cambridge University Press.

91 Berkes F. 1986. Marine inshore fishery management in Turkey. In: National Research

Council, editor. Proc. Conf. common Prop. Resour. Manag. Washington, D.C.: National Academy Press. p 63-83.

92 Fox ET. 2013. "Piratical schemes and contracts": Pirate articles and their society, 1660-1730. University of Exeter.

93 Leeson PT. 2009. The calculus of piratical consent: the myth of the myth of social contract. Public Choice 139:443-459.

94 Boyd R, Richerson PJ. 2002. Group beneficial norms can spread rapidly in a structured 
population. J Theor Biol 215:287-296.

95 Woodward C Van. 1974. The strange career of Jim Crow. Third Revi. New York: Oxford University Press.

96 Wright R. 2009. The evolution of god. New York: Little, Brown and Company. 97 Johnson SGB. 2018. Financial alchemists and financial shamans. Behav Brain Sci 41:e78. 98 Wootton D. 2015. The invention of science: A new history of the Scientific Revolution. Harper.

99 Hutton R. 2017. The witch: A history of fear, from ancient times ot the present. New Haven and London: Yale University Press.

100 Arthur WB. 2009. The nature of technology: What it is and how it evolves. Allen Lane. 101 Evans-Pritchard EE. 1937. Witchcraft, oracles, and magic among the Azande. Oxford, UK: Clarendon Press.

102 Singh M. 2021. Magic, explanations, and evil: The origins and design of witches and sorcerers. Curr Anthropol 62:2-29.

103 Acerbi A, Mesoudi A. 2015. If we are all cultural Darwinians what's the fuss about? Clarifying recent disagreements in the field of cultural evolution. Biol Philos Springer Netherlands. 30:481-503.

104 Singh M et al. 2021. Beyond social learning. Philos Trans R Soc B 376:20200050. 105 Knight J. 1992. Institutions and social conflict. Cambridge, UK: Cambridge University Press.

106 Alexander RD. 1979. Darwinism and human affairs. University of Washington Press. 107 Alexander RD. 1990. Epigenetic rules and Darwinian algorithms: The adaptive study of learning and development. Ethol Sociobiol 11:241-303.

108 Morin O. 2013. How portraits turned their eyes upon us: Visual preferences and demographic change in cultural evolution. Evol Hum Behav Elsevier Inc. 34:222-229.

109 Boyer P. 2001. Religion explained: The evolutionary origins of religious thought. New York: Basic Books.

110 Atran S. 1998. Folk biology and the anthropology of science: Cognitive universals and cultural particulars. Behav Brain Sci 21:547-609.

111 Mercier H, Boyer P. 2020. Truth-making institutions: From divination, ordeals and oaths to judicial torture and rules of evidence. Evol Hum Behav Elsevier Inc.

112 Miton $\mathrm{H}$ et al. 2015. Universal cognitive mechanisms explain the cultural success of bloodletting. Evol Hum Behav Elsevier Inc. 36:303-312.

113 Sperber D, Hirschfeld LA. 2004. The cognitive foundations of cultural stability and diversity. Trends Cogn Sci 8:40-46.

114 Barrett JL, Keil FC. 1996. Conceptualizing a nonnatural entity: Anthropomorphism in god concepts. Cogn Psychol 31:219-247.

115 Fitouchi L, Singh M. 2022. Supernatural punishment beliefs as cognitively compelling tools of social control. Curr Opin Psychol Elsevier Ltd. 44:252-257.

116 Norenzayan A et al. 2016. The cultural evolution of prosocial religions. Behav Brain Sci 39:e1.

117 Henrich J, Henrich N. 2010. The evolution of cultural adaptations: Fijian food taboos protect against dangerous marine toxins. Proc Biol Sci 277:3715-3724.

118 Singh M. 2018. Why is there shamanism? Developing the cultural evolutionary theory and addressing alternative accounts. Behav Brain Sci 41:e92. 
119 Warner WL. 1958. A black civilization: A social study of an Australian tribe. Harper \& Brothers. 


\section{Author Biography}

Manvir Singh is a research fellow at the Institute of Advanced Study in Toulouse. His research investigates why complex cultural traditions, such as music, hero stories, shamanism, and witchcraft beliefs, reliably emerge and exhibit recurrent features across human societies.

manvir.org 